Meta

Journal des traducteurs

Translators' Journal

\title{
Positionnement épistémologique de la traduction scientifique et technique, notion d'objectivité et implications pédagogiques
}

\section{Patricia Minacori}

Volume 50, numéro 4, décembre 2005

Pour une traductologie proactive - Actes

For a Proactive Translatology - Proceedings

Por una traductología proactiva - Actas

URI : https://id.erudit.org/iderudit/019871ar

DOI : https://doi.org/10.7202/019871ar

Aller au sommaire du numéro

Éditeur(s)

Les Presses de l'Université de Montréal

ISSN

0026-0452 (imprimé)

1492-1421 (numérique)

Découvrir la revue

Citer cet article

Minacori, P. (2005). Positionnement épistémologique de la traduction scientifique et technique, notion d'objectivité et implications pédagogiques. Meta, 50(4). https://doi.org/10.7202/019871ar
Résumé de l'article

La notion d'objectivité dans la réalisation d'une traduction scientifique et technique est un critère fondamental. Pour étudier cet élément de la démarche scientifique, nous positionnons l'opération traduisante sur un axe épistémologique. Cette opération est à la croisée des chemins entre positivisme et constructivisme, tout particulièrement lorsque l'on étudie deux éléments de sa méthode, la relecture et la révision. La rigueur dans la démarche d'un traducteur passe par des questionnements qui mènent à la connaissance. Il atteint un niveau de conscience suffisant pour mener à bien sa tâche et il applique ainsi une démarche proactive aux implications enseignables. 


\title{
Positionnement épistémologique de la traduction scientifique et technique, notion d'objectivité et implications pédagogiques
}

\author{
PATRICIA MINACORI \\ Université de Franche-Comté, Montbéliard, France \\ pminacori@wanadoo.fr
}

\begin{abstract}
RÉSUMÉ
La notion d'objectivité dans la réalisation d'une traduction scientifique et technique est un critère fondamental. Pour étudier cet élément de la démarche scientifique, nous positionnons l'opération traduisante sur un axe épistémologique. Cette opération est à la croisée des chemins entre positivisme et constructivisme, tout particulièrement lorsque l'on étudie deux éléments de sa méthode, la relecture et la révision. La rigueur dans la démarche d'un traducteur passe par des questionnements qui mènent à la connaissance. Il atteint un niveau de conscience suffisant pour mener à bien sa tâche et il applique ainsi une démarche proactive aux implications enseignables.
\end{abstract}

\begin{abstract}
The notion of objectivity for scientific and technical translation is a fundamental criterion. To study this element of a scientific procedure, translation could be positioned on an epistemological axis. We find translation between positivism and constructivism, particularly when rereading and revision are concerned. Questions can lead to knowledge through rigorous method. The translator then reaches a sufficient level of conscience and his mission can be carried out. He applies a proactive procedure, the implications of which can be taught
\end{abstract}

MOTS-CLÉS/KEYWORDS

traduction scientifique et technique, épistémologie, objectivité, relecture, révision

\section{INTRODUCTION}

«Prenez de la distance par rapport à votre traduction ». Voilà le conseil qu'un enseignant en traductologie peut avoir à donner à ses étudiants. Or, qu'est-ce que « prendre de la distance » par rapport à un texte scientifique ou technique dans le cadre d'une opération traduisante ?

Tout d'abord, il s'agit d'anticiper un événement bien connu des enseignants spécialisés en traductologie : les apprentis traducteurs s'accrochent à un texte de départ, à son enveloppe linguistique, sans essayer de pousser loin une pratique de compréhension de son sens. Dès lors, la formulation dans la langue d'arrivée n'est pas satisfaisante pour tout un ensemble de raisons. Prendre de la distance signifie surtout que le traducteur est amené à comprendre un texte dans tous ses aspects avant de pouvoir faire un travail de réexpression.

Face à ce constat, nous avons voulu mieux étudier la distanciation par rapport à un texte pour amener les étudiants traducteurs à tendre vers un caractère fondamental en traduction : l'objectivité. Cette notion est à la base de toute recherche scientifique.

Il est tout d'abord nécessaire d'étudier le positionnement épistémologique d'une discipline comme la traductologie. Cette étude peut se faire dans le cadre de deux grandes écoles de pensée occidentales : le positivisme et le constructivisme. Nous examinons leur position respective concernant l'étude de l'objet et du sujet de l'analyse.

Ensuite, l'analyse du réel permet d'en savoir plus sur l'objet et le sujet, afin d'en dégager certaines caractéristiques et mettre en évidence des éléments clés de la méthodologie. Pour ce faire, il est possible de dégager deux opérations fondamentales de méthodologie : la relecture et la 
révision d'une traduction que nous présentons à l'aide d'exemples de notre pratique

professionnelle, pour prendre en considération la notion d'objectivité.

Enfin, nous en tirons des conséquences enseignables.

Avant cela, il est utile de préciser notre cadre de réflexion.

\section{Positivisme et constructivisme}

La culture scientifique occidentale a eu pour habitude de se placer dans une doctrine positiviste selon laquelle l'objet à connaître est la réalité. Dans ce cadre, il existe un critère universel qui permet de légitimer les connaissances enseignables, celui de la vérité objective. Ainsi, il n'existe pas de relation entre le sujet et l'objet, les faits découlent de façon exclusive de l'observation et de l'expérimentation, ils peuvent ainsi être analysés de façon neutre et objective. D'après A. Comte (2002), une discipline scientifique, c'est-à-dire un champ de connaissances, se définit par son objet, le morceau de réalité objective qu'elle va décrire puis expliquer, en appliquant une méthode d'investigation. Cette dernière repose sur l'observation, la mesure et la répétitivité des faits. Aussi, peut-on expliquer les phénomènes et formuler des lois qui les régissent.

D'après J. L. Le Moigne (1994), le positivisme est régi par un certain nombre de principes :

1. il n'est pas possible de prendre en considération une proposition dont le contenu n'a, directement ou indirectement, aucune correspondance avec les faits constatés;

2. il n'existe aucune différence réelle entre l'essence et le fait;

3. tout jugement de valeur s'appuie sur une certitude scientifique;

4. toute connaissance ne doit se fonder que sur l'observation systématique des faits.

C'est pourquoi, J. L. Le Moigne (1994) propose le positionnement suivant :

L'épistémologie positiviste est une épistémologie de la vérification : elle ambitionne de s'ordonner sur la production d'énoncés vrais parce que vérifiés à la fois par le raisonnement démonstratif et l'observation empirique (Le Moigne 1994, T. $1:$ 123).

Or, des voix se sont élevées dans un souci de remettre en perspective la thèse selon laquelle objet et sujet sont indépendants. Certains courants philosophiques ont en effet réfuté l'existence d'un monde réel extérieur au sujet, et notamment Kant : « Le monde est inconnaissable et ma connaissance est phénoménologique » (Kant $2000: 251$ ).

Sans entrer dans un débat de spécialistes, qui voudrait reconnaitre une filiation du constructivisme à certains (Hume, voire, d'après Le Moigne (1994) Protagoras, Aristote ou Pyrrhon ), ce courant de pensée s'est lentement élaboré et il a trouvé son plein épanouissement dans la première moitié du XX $X^{\mathrm{e}}$ siècle. En effet, R. Bachelard (1999) va contribuer à ébranler la construction positiviste dans une phrase devenue fort célèbre : «Rien n'est donné. Tout est construit » (Bachelard, 1999 : 14) .

Puis, J. Piaget, souvent cité par les spécialistes comme l'un des pères fondateurs du constructivisme, va émettre l'idée selon laquelle :

L'intelligence (et donc l'action de connaître) ne débute ainsi ni par la connaissance du moi, ni par celle des choses comme telles, mais par celle de leur interaction; c'est en s'orientant simultanément vers les deux pôles de cette interaction qu'elle organise le monde en s'organisant elle-même (Piaget 1967 : 311).

D'après J. Piaget, il existe une interaction entre « la connaissance du moi » et celle « des choses » et, avec cette interaction, l'acte de connaître un objet et celui de se connaître sont inséparables. Il existe ainsi une interaction entre l'objet ou le phénomène à connaître et le sujet connaissant. Ce qui revient à dire :

Le réel connaissable est un réel en activité qu'expérimente le sujet et [...] ce sujet se construit, par des représentations symboliques, cette connaissance de son expérience du réel (Le Moigne 1995: 72). 
Le constructivisme postule ainsi que l'on ne peut connaître la réalité en soi mais seulement ses représentations. Il n'est plus question de se demander s'il existe ou s'il n'existe pas un réel connaissable ou inconnaissable. En revanche, le sujet construit sa propre connaissance du réel :

Le réel existant et connaissable peut être construit par ses observateurs qui sont dès lors ses constructeurs (on dira plus volontiers ses modélisateurs) (Le Moigne 1995 : 40).

A partir de ce fondement théorique nous plaçons la traductologie dans un cadre épistémologique.

\section{Le positionnement épistémologique de la traductologie}

Nous venons de rapidement fixer un environnement épistémologique avec les conséquences citées plus haut :

- dans le premier, le positivisme, le sujet et l'objet sont séparés, l'objectivité est de mise; - dans le second, le constructivisme, sujet et objet sont en interaction et la subjectivité prime.

Quelles sont les implications en traductologie?

\subsection{Préliminaires}

En traductologie, le sujet est le traducteur et l'objet ou le réel est représenté par le texte, ou plus précisément les textes, celui exprimé dans une langue de départ et celui exprimé dans la langue du traducteur, en général, sa langue maternelle. Nous entendons par texte :

Toute unité de production verbale écrite, constituée d'un début, d'un milieu et d'une fin, véhiculant un message linguistique organisé, dont la principale fonction est d'être vecteur de sens, tendant à produire sur son destinataire un effet de cohérence, dans un objectif de communication (Minacori-Vibert 2001 : 97).

Or, l'opération traduisante se reflète dans le passage entre ces deux textes, dans un objectif très précis, celle de donner la primauté au texte d'arrivée, à l'issue d'un ensemble de procédures complexes, au cours desquelles le traducteur comprend un message pour réexprimer cette compréhension. Il ne s'agit pas de récuser en bloc l'importance du texte de la langue de départ, comme il le sera souligné plus loin, mais de voir qu'une fois que le traducteur a saisi tout son contenu, après avoir mis en œuvre une démarche de compréhension poussée très loin, dans le plein respect du vouloir dire de l'auteur mais aussi en prenant en considération le destinataire de la traduction, le traducteur se focalise sur ce dernier. Comprendre un texte d'origine signifie : avoir les connaissances lexicales, terminologiques, notionnelles, comprendre tout le déroulement du fil logique du texte, ses implicites, son intention, son objectif. Comprendre c'est bien «prendre avec » au sens étymologique du terme. Comprendre, c'est aussi construire une représentation cohérente en mémoire (Blanc, Brouillet 2003). Enfin, comprendre c'est aussi aller vers le sens voulu par l'auteur en appliquant une démarche qui n'est pas autocentrée autour du traducteur, et ce, dans la rigueur et l'écoute de ce que veut dire l'auteur, d'où notre volonté de mettre en évidence un critère d'objectivité qui se dégage de la phase de compréhension.

L’objectivité étant la :

qualité de ce qui donne une représentation fidèle d'un objet. Qualité de ce qui est exempt de partialité, de préjugés. Attitude d'une personne impartiale objective (Robert :1512).

Nous nous plaçons en effet dans une perspective professionnelle, dans laquelle, la traduction ne figure pas en vis-à-vis du texte d'origine, comme cela pourrait être le cas dans un 
secteur d'activité comme la publication d'œuvres littéraires bilingues, par exemple. Nous ne sommes pas dans une optique comparatiste qui consisterait à voir comment une langue découpe la réalité par rapport à une autre langue, pour en tirer des conséquences enseignables. En effet, il ne s'agit pas de mettre en évidence un système linguistique par rapport à un autre, dans la similitude, dans la différence et dans tous les degrés possibles entre ces deux états.

En revanche, lors de l'opération traduisante, un traducteur va appréhender le sens d'un texte pour recréer un autre texte, dans sa langue maternelle, dans une pratique de rigueur, dans le respect de l'intention et de la logique de l'auteur. Il a été détaillé ailleurs les connaissances et le savoir-faire de base du traducteur (Minacori-Vibert 1999, c). Il importe alors, d'analyser l'objet de la traductologie.

\subsection{L'objet de la traductologie}

Pour préciser l'objet d'une discipline, il est possible de se fonder sur la définition suivante :

L'objet d'une discipline est soit son intention, son dessein, ou son objectif, soit sa manière propre à quoi s'applique son étude spécifique (Canguillem 1971 : 7).

La deuxième partie de cette définition « la manière propre à quoi s'applique son étude spécifique », offre des perspectives intéressantes pour étudier l'objet de la traductologie et tout particulièrement ses opérations de contrôle. En effet, dès l'instant qu'une traduction est indissociable d'un traducteur, il existe un danger très réel ou du moins une difficulté : tendre vers l'objectivité, en terme clair, produire une traduction à la qualité irréprochable, c'est-à-dire savoir mettre en évidence d'éventuelles difficultés de compréhension et d'expression et les résoudre.

Les opérations de contrôle d'une traduction se manifestent, d'une part, dans un processus de relecture et, d'autre part, dans une pratique de révision.

\subsubsection{Les opérations de relecture}

Une fois le texte traduit, le traducteur met en œuvre un processus de relecture où il va effectuer des allers-retours entre le texte original et sa traduction à des fins de contrôle. La relecture est une opération qui peut se détailler en trois phases principales.

\subsubsection{Les trois phases de la relecture}

La relecture d'une traduction s'apparente à une vérification, tout d'abord, du respect du sens voulu par un auteur dans une pratique de rigueur et de précision, du respect des notions et de la terminologie scientifique et technique de la langue d'arrivée et enfin du respect de la morphosyntaxe de cette dernière. En effet, si l'on détaille ces opérations on peut dégager les trois étapes suivantes :

- premièrement, il est important de mettre en parallèle le texte exprimé dans la langue de départ et celui exprimé dans la langue de la traduction pour voir si tout les messages exprimés dans le premier figurent bien dans le second. Cette première opération permet, notamment, de vérifier qu'aucun membre de phrase, qu'aucune phrase, voire paragraphe ou page n'ont été omis, si tous les schémas, figures, tableaux ont été traduits, etc. Cette première phase permet de vérifier que le fil logique de l'auteur a été respecté par le traducteur, qu'aucun élément du raisonnement n'a été oublié; - deuxièmement, il est fondamental de relire la traduction proprement dite. Il est conseillé de relire le texte traduit seul, pour se détacher de l'enveloppe linguistique du texte original, afin de concentrer son attention sur la qualité de la langue de la traduction. Pour les étudiants c'est une phase fondamentale au cours de laquelle ils prendront garde à ne pas recourir aux procédures de calques selon la définition de M. Pergnier (1989): 
« influence d'un système linguistique sur l'autre aboutissant à un résultat hybride » (Pergnier 1989 : 23). Le recours aux calques est en effet parasitaire et fortement dommageable pour la qualité du français (Minacori 2000);

- troisièmement, il s'agit de relire la traduction pour en vérifier la qualité orthographique, syntaxique, etc. En effet, puisque les correcteurs orthographiques ne sont pas (encore) omniscients, cette phase de relecture de la forme est tout à fait nécessaire. Relire à la fois le fond et la forme d'une traduction nécessite une grande concentration et la mémoire de travail d'un traducteur se trouve parfois débordée. C'est pourquoi, nous préconisons à nos étudiants d'avoir recours à cette troisième phase de relecture.

Trop souvent, la relecture n'est pas appréciée à sa juste valeur. Il est vrai que les temps de relecture sont longs et que les traducteurs professionnels doivent travailler dans l'urgence. Néanmoins, une relecture dans laquelle ces trois phases n'ont pas été prises en considération est la porte ouverte à des erreurs qui peuvent entacher le caractère professionnel d'une traduction, comme nous allons le démontrer maintenant.

\subsubsection{Deux exemples de relecture}

Une organisation internationale nous a demandé de réviser une traduction sur l'électricité statique et les risques encourus par le personnel de sites industriels. Il s'agit de vérifier le savoir-faire d'un traducteur et dans un premier temps de vérifier si les opérations de relecture ont bien été réalisées.

On pouvait lire, dans le texte original, les passages suivants :

«The phenomenon of generation of static electricity by friction (triboelectrification) has been known for thousands of years. Contact between two materials is sufficient to induce electrification... »

«... In practice, electrification occurs only in liquids with resistivity greater than ... »

Pour faciliter notre démonstration, nous avons écrit en caractères gras les points révisés par nos soins.

Ces deux paragraphes ont été traduits de la façon suivante :

«Le phénomène de production d'électricité statique par friction (triboélectricité) est connu depuis des milliers d'années. Le contact entre deux matériaux suffit pour induire une électrisation.... »

« ... En pratique, l'électrifisation se produit uniquement dans les liquides dont la résistivité, etc. »

Dans cet exemple, la deuxième phase de relecture, qui consiste à détailler la traduction sans l'original pour focaliser son attention sur le texte traduit a fait défaut. En effet, la traduction du terme anglais « electrification » est rendu, dans un premier temps, par «électrisation » et, dans une second temps, par « électrifisation ».

On remarque également que le fait d'utiliser en français « électrifisation » laisse à penser que les notions de base en électricité ne sont pas acquises.

La relecture s'apparente à un travail d'enquête et cette notion mérite quelques explications. En effet, l'enquête se déroule tout particulièrement lorsque un traducteur est confronté à une difficulté de compréhension dans le texte de la langue de départ. Il fait alors appel à tout un réseau de connaissances linguistiques, notionnelles, de raisonnement logique qui vont lui permettre de rechercher des indices dans le texte lui-même, dans le cotexte, c'est-à-dire les éléments textuels qui viennent immédiatement avant ou après le passage concerné, plus loin dans le texte et, enfin, dans d'autres textes, qui constituent la documentation au sens large du terme. Cette dernière comprend un ensemble de données terminologiques, phraséologiques et notionnelles dans lesquelles le traducteur crée des liens hypertextuels. Enfin, il peut avoir recours à la consultation d'experts du domaine concerné. 
Ces données sont en phase avec les recherches à établir, c'est-à-dire que, face à une multitude de données qu'il est possible de traiter, le traducteur sait axer au mieux ses recherches pour résoudre la difficulté rencontrée. Il va ainsi collecter des indices permettant de formuler des hypothèses qu'il va valider à la lumière de son propre raisonnement, et de ses propres connaissances et aussi en fonction des outils documentaires utilisés. Une fois son hypothèse formulée, il la vérifie pour asseoir sa compréhension, et, faire un choix de formulation. En effet, sans une recherche approfondie concernant la compréhension d'un texte, il ne peut y avoir expression dans une autre langue.

Venons-en à un deuxième exemple. Dans le texte déjà cité sur l'électricité statique, on pouvait lire dans le texte original :

"In explosive atmospheres, it is the environment itself that is sensitive to electrostatic discharges, and discharges may result in ignition or explosion. Protection in these cases consists of replacing the air, either with a gas mixture whose oxygen content is less than the lower explosive limit, or with an inert gas, such as nitrogen ».

Le traducteur a proposé pour ce passage :

« Dans les atmosphères explosives, c'est l'environnement lui-même qui est sensible aux décharges électrostatiques et celles-ci peuvent provoquer une incendie ou une explosion. Dans ce cas, la protection consiste à remplacer l'air par un mélange gazeux dont la teneur en oxygène est plus faible que la limite d'explosibilité inférieure ou par un gaz inerte tel que le nitrogène. »

Cet exemple illustre que la troisième phase de relecture n'a pas été appliquée par le traducteur, puisque l'on peut lire « une incendie ». Enfin, et surtout, les connaissance du traducteur concernant les gaz inertes font défaut, car celui-ci s'est, à nouveau, laissé porté par un calque délétère, sans mettre en œuvre une distanciation nécessaire à la qualité de son texte. En effet, l'équivalent en français de « nitrogène » est bien « azote ».

Le texte révisé que nous avons proposé est le suivant :

« En atmosphère explosive, c'est l'environnement lui-même qui est sensible aux décharges électrostatiques et celles-ci peuvent provoquer un incendie ou une explosion. Dans ce cas, pour se protéger, il convient de remplacer l'air par un mélange gazeux dont la teneur en oxygène est plus faible que la limite d'explosibilité0 inférieure ou par un gaz inerte tel que l'azote. »

En conséquence, la relecture s'apparente à un travail d'enquête dans lequel le traducteur s'implique et sait prendre une distance suffisante pour remettre son travail en cause et tendre ainsi vers une qualité professionnelle. Si l'on pousse encore plus loin ce raisonnement, on arrive alors à un deuxième type de travail d'enquête, la révision. Elle fait partie de ce cheminement, elle s'apparente à une phase de relecture, non plus réalisée par le traducteur lui-même, mais par un auteur différent dans la chaîne de communication.

\subsubsection{La révision}

Dans certains pratiques professionnelles, comme la traduction dans les organisations internationales, il est fait systématiquement recours à des réviseurs, qui sont des traducteurs expérimentés.

Un réviseur a des connaissances et un savoir-faire. Il a une bonne connaissance du domaine, des textes, objets de la révision : il connaît la terminologie, la phraséologie et il a aussi les connaissances notionnelles du domaine concerné. Il sait aussi constituer des opérations de contrôle avec des supports papier ou en ligne, comme les manuels, les dictionnaires et les encyclopédies et, s'il y a lieu, les contacts avec un ou plusieurs experts ont été établis, pour aboutir à une parfaite compréhension du domaine à traduire. Aussi, tout le travail d'enquête est-il connu du réviseur : les 
démarches de recherches notionnelles et terminologiques sont acquises par une longue pratique du métier.

Par ailleurs, il n'est pas un spécialiste d'une technique spécifique mais d'une méthode précise. Il a un savoir-faire différent du technicien, de l'ingénieur et du scientifique. On ne lui demande pas de savoir exécuter des tâches de fonderie ou d'emboutissage lorsque le texte à réviser traite du secteur automobile, mais d'avoir une connaissance approfondie du domaine concerné, de savoir raisonner sur un texte, collecter des informations nécessaires pour mener à bien le rôle assigné. Il peut alors repérer, dans la traduction, les difficultés éprouvées par le traducteur. Le réviseur applique donc une méthode qui se veut empreinte d'objectivité.

On peut illustrer la distanciation nécessaire à la qualité d'un texte traduit par deux exemples.

Reprenons le texte sur l'électricité statique : il faisait référence à une norme européenne portant sur la distribution électrique :

«The European Standard EN 50110-1, Operation of Electrical Installations, prepared by CENELEC Task Force 63-3 is the basic document that applies to the operation of and work activities on, with or near electrical installations..... The standard applies to installations designed for the generation, transmission, conversion, distribution and use of electrical power, and operating at commonly encountered voltage levels. Although typical installations operate at low voltages, the standard also applies to extra-low and high-voltage installations... »

Dans sa démarche documentaire, le traducteur peut, très utilement, rechercher si cette norme européenne EN 50110-1 (1994) a été traduite en français. Si la réponse est affirmative, il peut chercher à se procurer la traduction en français.

En effet, le début du texte à traduire est :

«The European Standard EN 50110-1, Operation of Electrical Installations, prepared by CENELEC Task Force 63-3 is the basic document that applies to the operation of and work activities on, with or near electrical installations »

Le traducteur a proposé :

« La norme européenne EN 50110-1 "Fonctionnement des installations électriques (1994)", préparée par le groupe de travail 63-3 DU CENELEC, constitue le document de base réglementant le fonctionnement des installations électriques et les travaux effectués sur, avec ou à proximité de ces installations. »

Or, le premier paragraphe de la dite norme en français EN 50110-1 (1994) mentionne :

«Cette norme s'applique à l'exploitation des installations électriques et aux travaux, sur, avec ou dans l'environnement des installations électriques ».

À l'évidence, le traducteur n'a pas eu accès à cette norme. En tout état de cause, il s'en suit une traduction que l'on peut qualifier de «non aboutie ».

C'est pourquoi, après avoir pris connaissance de la norme en français, il est possible de réviser le texte en français comme suit :

«La norme européenne EN 50110-1 (1994) "Exploitation des installations électriques" préparée par le groupe de travail 63-3 DU CENELEC, constitue le document de base s'appliquant à

l'exploitation des installations électriques et aux travaux effectués sur, avec ou dans

l'environnement de ces installations. »

Il semble logique, dans ce cas, d'utiliser la terminologie de la norme traduite en français. Avant d'en examiner les raisons, passons à l'analyse du second exemple. 
L'original de la traduction est le suivant :

«The standard applies to installations designed for the generation, transmission, conversion, distribution and use of electrical power, and operating at commonly encountered voltage levels. Although typical installations operate at low voltages, the standard also applies to extra-low and high-voltage installations... »

La traduction proposée pour ce passage est :

«Cette norme s'applique aux installations qui ont été conçues pour la génération, la transmission, la conversion, la distribution et l'utilisation de la puissance électrique et qui fonctionnent à un niveau de tension fréquemment utilisé. Bien que les installations fonctionnent souvent à basse tension, la norme s'applique également aux installations à très basse ou à haute tension. »

Dans une premier temps, nous avons révisé ce texte comme suit :

«Cette norme s'applique aux installations pour la production, le transport, la conversion, la distribution et l'utilisation de l'énergie électrique, à un niveau de tension fréquemment utilisé. Bien que ces installations fonctionnent souvent à basse tension, cette norme s'applique également aux installations à très basse et à haute tension. »

Puis face à ce qui nous semblait une incohérence, celle d'appliquer une norme aux installations à très basse tension, d'une part, et celles à haute tension, d'autre part, nous avons recherché dans le document de référence, une réponse. Nous avons pu lire le passage suivant :

«Cette norme s'applique à l'exploitation des installations électriques et aux travaux sur, avec ou dans l'environnement des installations électriques. Ces installations fonctionnent à des niveaux de tension allant de la très basse tension comprise et jusques et y compris la haute tension.

Ce dernier terme inclut les niveaux correspondant aux appellations de moyenne et de très haute tension. » (EN 50110-1 : 1994).

Or, il existe manifestement une information donnée dans la norme traduite en français dans le passage « ...à des niveaux de tension allant de la très basse tension comprise et jusques et $\mathbf{y}$ compris la haute tension. », précision qui varie sensiblement dans l'information donnée dans le texte à traduire en anglais «the standard also applies to extra-low and high-voltage installations... »

Il aurait aussi été possible de traduire : « allant de la très basse à la haute tension comprises », formulation moins lourde que celle que nous avons choisie. En fait, nous avons voulu privilégier la terminologie utilisée dans la norme européenne.

La rédaction de ce type de normes est soumise à un ensemble de procédures longues et précises qui peuvent prendre entre 2 et 5 ans, d'après les spécialistes. Au cours de nombreuses réunions, un ensemble d'experts avalisent la terminologie des normes ainsi que leur traduction. C'est pourquoi, les informations contenues dans des normes ont un coefficient de fiabilité supérieur à celui des informations contenues dans un article, si rigoureux et précis soit-il. D'où une alternative devant laquelle se trouve le traducteur : soit considérer ce coefficient de fiabilité, et maintenir le sens présent dans la norme, soit respecter le message de l'auteur de l'article. A partir du moment où le traducteur a compris le message de l'auteur et aussi lorsqu'il est en mesure de voir que ce message n'est pas complètement en phase avec les informations auxquelles il est fait référence, le traducteur est devant un choix. La résolution de cette difficulté peut notamment passer par le contact avec l'auteur de l'article pour trouver une solution. C'est bien ce que nous avons fait et l'auteur a modifié son texte pour respecter l'esprit de la norme dont il était question.

Il ressort de cet exemple, l'importance de l'esprit critique qu'un réviseur peut exercer, et l'illustration d'un degré ultime de la distanciation, dans le cas où une incohérence survient entre le texte à traduire et dans le cas présent, un texte de référence. 
En conséquence, nous avons vu que le réel en traductologie est constitué par des textes. La méthodologie est un aspect par lequel on peut aborder l'étude de son objet, et plus particulièrement l'analyse fine des opérations de relecture et de révision. Un traducteur comprend et traduit un texte exprimé dans une langue étrangère, recréant un autre texte, dans sa langue maternelle, il relit ce texte dans le cadre d'une phase de finalisation de la traduction, puis un réviseur met en œuvre des procédures de contrôle d'une traduction ; il existe alors une interaction entre le réel et objet. En effet, il ne semble pas pouvoir dissocier l'un de l'autre, puisque le traducteur (ou le réviseur) part d'un texte, pour aboutir à un autre texte, il part d'une œuvre de création intellectuelle pour aboutir à une autre œuvre du même type, dans une pratique de rigueur, qui se fait jour dans les opérations de contrôle comme la relecture et la révision.

Si l'on passe l'opération traduisante à la loupe, il existe une interaction incessante et intrinsèque entre sujet et objet. Or, si tel est le cas, ses observateurs deviennent alors ses modélisateurs. C'est pourquoi, en se fondant sur ces divers éléments, il est possible d'avancer que la traductologie peut se positionner sur un axe constructiviste.

Or, en sciences humaines, le critère d'objectivité semble d'un point de vue théorique impossible à appliquer dans le cadre du constructivisme puisque l'observateur n'est jamais étranger aux conditions observées. En revanche, on pourrait penser que dans certains domaines, il règne une objectivité réelle. Par exemple, les mathématiques pourraient être considérées comme le symbole de l'objectivité. Cependant, si l'on analyse les données mathématiques d'un point de vue historique, une vérité mathématique est partagée par les membres d'un groupe à un moment donné. Le sens donné à une formule est forgé par celui qui crée ces symboles, jusqu'à ce que un membre d'un autre groupe et à un autre moment, mette cette vérité en question.

En effet :

La science elle-même est loin d'être tout entière scientifique, au sens où ce mot qualifie un savoir parfaitement objectif, ne laissant plus aucune place à la contestation (Blanché 1972 : 123).

L'objectivité semble donc douée d'une certaine labilité, puisque tout savoir n'est pas parfaitement objectif. Or, nous venons de voir que si, dans une opération traduisante, elle n'est pas prise en considération, le travail réalisé n'est pas professionnel.

Traduire c'est d'abord comprendre un texte en appliquant une démarche scientifique dans laquelle l'objectivité est présente. Dans cette phase, cette opération se trouve à la croisée des chemins entre positivisme et constructivisme. Traduire c'est ensuite réexprimer un texte dans la langue du traducteur et c'est là qu'intervient sa subjectivité car il va exprimer sa compréhension avec ses propres moyens linguistiques, dans le respect de la formulation de sa langue maternelle. Dans cette seconde phase, cette opération se positionne sur un axe constructiviste.

Une opération traduisante à l'issue de laquelle un traducteur prend une distance nécessaire tout particulièrement dans la phase de compréhension, permet alors de tendre vers un critère d'objectivité, et partant, vers une qualité professionnelle indéniable. Lorsque la logique de l'enquête est poussée fort loin, comme dans certains pratiques professionnelles, dans lesquelles les textes sont systématiquement soumis à révision, il se dégage de ces pratiques un souci d'objectivité et un gage de professionnalisme.

Cet enseignement est fondamental pour les étudiants traducteurs et pour les traducteurs euxmême. L'opération traduisante ne peut se faire que dans une parfaite pratique de compréhension, lorsque les questions que l'on est en droit de se poser face à un texte sont résolues. En effet : « Toute connaissance est une réponse à une question » (Bachelard 1999 : 14).

Il ressort de tous ces exemples que l'objectivité c'est bien la distance par rapport à un texte. Elle naît d'un questionnement incessant pour saisir la compréhension dans tous ses aspects.

Cette démonstration trouve des applications pédagogiques dans l'enseignement de la traduction scientifique et technique. 


\section{Les implications pédagogiques}

La question centrale à laquelle nous souhaitons répondre est la suivante : qu'est-ce qui permet de prendre de la distance par rapport à une traduction ? En d'autres termes, pourquoi un traducteur écrit en français « nitrogène » à la place d'azote, pourquoi il a eu recours, dans un premier temps à « électrifisation » puis dans un second à «électrification »? Quelles conséquences en tirer pour les étudiants traducteurs?

Dans ces deux exemples, si l'on analyse la typologie de l'erreur, il semble que le traducteur a simplement eu recours à un calque. Si c'est bien le cas, cela veut dire que ses connaissances ne lui ont pas permis de rétablir la vérité car il n'a pas eu conscience de cette difficulté de compréhension. Pour le premier exemple de «nitrogène », il se peut qu'il ait vaguement eu le souvenir que ce terme devait exister, ou alors établir un lien avec oxygène, mais sans pousser plus loin sa recherche pour vérifier ses impressions. Or, «nitrogène » est un terme vieilli, et unanimement remplacé de nos jours par «azote » en français. Dans ces deux cas, il semble que le degré de conscience ait été quasi nul. Aucune distance n'a été prise sur le texte, et ce point de vue appartient à un individu seul, de façon autocentrée : on est proche du mythe de la caverne de Platon. On pourrait qualifier cette étape de « degré 0 de conscience », sur une échelle qui irait de 0 à 5 , par exemple.

En cherchant quelles étapes mènent à une traduction aboutie, il est possible d'en dégager au moins deux :

- le traducteur sait de façon consciente que ses connaissances sur le terme anglais de « nitrogen » ne sont pas assez complètes, il n'a pas les idées très claires sur ce terme, ou il peut avoir une idée selon laquelle ce terme pourrait être l'équivalent d' "azote » en français. Dans ces cas, il va effecteur des recherches et l'état qui caractérise cette « idée » situer sur une échelle de 1 à 4, sur le degré de conscience, en fonction de son pourrait se ou moins vague. C'est un état de connaissance passive de la notion d'azote; caractère plus

- lorsqu'un traducteur rencontre le terme de «nitrogen » dans un texte en anglais, il sait de façon consciente qu'il s'agit d'une molécule qui, en français s'appelle désormais « azote », dont le numéro atomique est 7 , qui entre dans $4 / 5$ de la composition de

l'atmosphère, et dans les tissus vivants comme les protéines, etc. Dans ce cas particulier, il a une connaissance active concernant cette molécule, il ne ressent pas le besoin de poursuivre plus loin ses recherches : c'est le degré 5 de conscience.

Dans l'analyse de ces trois procédures, on passe d'un degré 0 de conscience, que l'on peut aussi nommer degré de non conscience, à deux autres degrés : l'un permet au traducteur de penser que ses connaissances ne sont pas suffisantes et l'autre lui permet d'activer, d'appliquer ses connaissances concernant cette molécule.

On voit alors que le degré 0 de conscience est le plus dangereux pour la qualité d'une traduction : il n'y a aucune distance par rapport au texte, le traducteur ne se pose aucune question, il semble sur un rail. Cette attitude se fait au détriment de la qualité du texte final lui-même. Sa démarche n'a rien de scientifique puisque la subjectivité occupe tout le terrain.

Du degré 1 au degré 5 de conscience, le danger semble écarté, car le traducteur prend la distance nécessaire par rapport à la traduction. S'il se pose une question, il va chercher à y répondre en mettant en action une recherche adéquate. S'il a clairement le sentiment que ses connaissances sont nécessaires et suffisantes, il n'a aucune recherche à effectuer. Dans ces deux cas, le traducteur fait preuve d'objectivité.

Il est possible de dégager de ces exemples et de notre expérience d'enseignante de traduction, une démarche proactive pour aider les étudiants à progresser, notamment ceux de première ou de deuxième année à l'université. En effet, dans un premier temps nous avons fait une typologie des erreurs de traduction les plus fréquentes, repérées lors des cours mais aussi des examens. Lorsqu'un étudiant propose une traduction en cours, il est facile de mettre en évidence ce qui fait défaut dans sa compréhension. Celles-ci sont de plusieurs types : lexicales, morphosyntaxiques, notionnelles, terminologiques, etc. Or, si un étudiant sait mettre en évidence, de façon active, d'où vient son incompréhension, il peut alors agir très précisément pour pallier cette situation (Minacori 1999 a et b). 
Il s'agit alors de faire réagir l'étudiant sur son texte, de lui faire prendre conscience de son erreur, de l'analyser et donc de réagir pour modifier sa proposition de traduction. Cette démarche devrait pouvoir s'appliquer à l'avenir : comprendre que l'on ne comprend pas et agir en conséquence, pour répliquer cette méthode. Nous essayons d'entrainer les étudiants à verbaliser leur non compréhension et à réagir et nous avons remarqué que l'on n'habitue pas assez les étudiants à analyser la source de leurs incompréhensions et de résoudre ces dernières.

En revanche, il est difficile de pouvoir anticiper le passage du degré 0 au degré 1 , lors de la prise de conscience d'une difficulté de compréhension. Pour l'instant nous n'avons pas d'éléments de réponse. Notre constat est le suivant : en première année d'études supérieures, les étudiants qui éprouvent le plus de difficultés ont une mémoire de travail qui semble débordée par le manque de vocabulaire, de notions grammaticales, de logique, etc. Est-ce à dire que nous n'avons pas d'action à mener pour améliorer leurs prestations ? Nous pensons, au contraire, que les cours de traduction sont un formidable tremplin pour exercer leur compréhension des textes exprimés dans une langue étrangère et progresser dans cette langue en fournissant des efforts adéquats c'est-à-dire quotidiens.

Cette démarche proactive n'est pas toujours facilement applicable, car, même en année de Master, certains étudiants n'arrivent pas à passer au crible certaines difficultés de traduction, car certains glissent sur un concept qui n'est pas connu, car il contient un certain niveau de transparence linguistique.

Par exemple, nous avons proposé à des étudiants de Master Lettres Langues et Commerce Electronique de 1'Université de Franche-Comté, à Montbéliard la traduction d'un texte sur les conditions générales régissant le site du Metropolitan Museum of Art de New-York (http://www.museum.org/copyright.htm) Dans la partie «Terms and Conditions » et plus précisément dans le paragraphe 2 on peut lire :

«2. The materials are made available for limited non-commercial, educational, and personal use only, or for fair use in the United States copyright laws ... »

Certains étudiants ont eu l'impression de comprendre la collocation «fair use » qu'ils ont traduite par « usage équitable », « usage loyal », etc. sans se lancer dans des recherches. D' autres ont tout de suite reconnu la notion juridique de «fair use » énoncé dans le titre $17 \mathrm{du}$ Code des Etats-Unis, section 107. D'autres encore, ont été alerté par la mention de « as defined in the United States copyright laws » et ils ont fait les démarches nécessaires pour se rendre compte que cette notion correspond « au droit de citation » en France.

A partir de ce dernier exemple, nous soulignons qu'il importe d'enseigner la pratique de la relecture et de la révision dans tout cursus universitaire dont la finalité est la formation de traducteurs scientifiques et techniques.

\section{CONCLUSION}

L'invariant qui nous semble important de retenir est le suivant : traduire signifie avoir un esprit sans cesse en alerte, sur le chemin qui mène au sens. Il s' agit de connaître et aussi de reconnaître les notions techniques, scientifiques, juridiques, même celles qui semblent transparentes sur le plan linguistique, au risque d'entacher la qualité de la traduction. Traduire c'est d'abord comprendre un texte et appliquer un critère d'objectivité emprunté à la démarche scientifique du positivisme. En effet, si l'on regarde les critères recherchés dans toute traduction aboutie, on remarque qu'ils reflètent l'esprit d'observation, l'esprit critique, l'impartialité et la rigueur. Traduire c'est aussi exprimer cette compréhension, avec une subjectivité prudente.

Comprendre procède d'un savoir-faire méthodologique car il faut pousser loin cette faculté de questionnement, cet esprit critique qu'il est plus difficile d'atteindre dans la relecture de son propre texte que dans la révision d'un texte traduit par une tierce personne. Néanmoins, une pratique de compréhension d'un texte, peut se faire par la méthode de la relecture, en habituant les étudiants à identifier leurs difficultés de compréhension et à résoudre ces dernières. Cette formation attend 
vraiment une complétude lorsque les activités qui président à la révision d'un texte sont aussi acquises par les étudiants.

Former les étudiants traducteurs à une bonne pratique de la relecture et de la révision est un minimum. Cela reflète une démarche proactive, qui peut avoir un effet boule de neige : plus on leur apprend à se poser les bonnes questions sur un texte et mieux ils appliqueront cette méthode. Pour l'instant, nous n'avons pas de solution miracle pour que les étudiants qui sont au niveau 0 de conscience passent au niveau 1. C'est tout le nœud gordien de la compréhension humaine. C'est un grand défi, qui s'offre à nous et que nous tentons de cerner peu à peu. Étudier ce qui se passe dans l'esprit humain pour comprendre un texte dans sa langue maternelle et dans une langue étrangère, notamment en ce qui concerne les différents types de mémoires, offre un vaste champ d'étude.

\section{RÉFÉRENCES}

BACHELARD, G. (1999) : Le nouvel esprit scientifique, Paris, PUF.

Blanc, N. et D. Brouillet (2003) : Mémoire et compréhension, Lire pour comprendre, Paris, Editions In Press. Blanche, R. (1972) : L'épistémologie, Paris, PUF, Coll. Que sais-je, n 1475.

CANGuillem, G. (éd) (1971) : Introduction à l'histoire des sciences, vol 2, Paris, Hachette.

Conte, A. (2002 ) : Discours sur l'esprit scientifique, Paris, Vrin, Coll. Bibliothèque des textes scientifiques.

KAnT, E. (2000) : Critique de la faculté de juger, Paris, GF Flammarion.

Le Moigne, J.-L. (1995) : Les épistémologies constructivistes, Paris, PUF, Coll. Que sais-je, n²969.

Le Moigne, J.-L. (1994) : Le constructivisme, 2 tomes, Paris, ESF Editeurs.

MiNACORI, P. (2001) : «La traduction scientifique et technique : la notion de texte et ses implications pédagogiques », La Revue des Lettres et de Traduction, Université de Saint Esprit de Jounieh, Liban, n 7, p. 87-110.

MinACORI, P. (2000) : «Les prérequis pour le recrutement d'étudiants traducteurs », Maison du Dictionnaire, Actes du Colloque international Rennes 2 Formation des traducteurs (24-25 septembre 1999), Éd. Daniel Gouadec, p 6065.

MinACORI, P. (1999a) : «La compréhension en traduction : aspects théoriques », French Studies in Southern Africa, Prétoria, $\mathrm{n}^{\circ} 28$, p. 77-91.

MinACORI, P. (1999b) : «La compréhension en traduction : aspects professionnels et pédagogiques », French Studies in Southern Africa, Prétoria, n² 28, p. 92-108.

MINACORI, P. (1999c) : «La compréhension en traduction : aspects pratiques pour les étudiants traducteurs francophones et non francophones », French Studies in Southern Africa, Prétoria, n² 28, p. 109-132.

Piaget, J. (éd.) (1967) : Logique et connaissance scientifique, Paris, Gallimard, Encyclopédie de la Pléïade. Pergnier, M. (1989) : Les anglicismes, Paris, Presses Universitaires de France.

Rey-Debove, J. et A. Rey (dir.) (2002) : Le nouveau petit Robert 1. Dictionnaire alphabétique et analogique de la langue française, Paris, Dictionnaires Le Robert. 